\title{
REVIEW
}

\section{Autoimmune Pathogenesis of Chagas Heart Disease}

\section{Looking Back, Looking Ahead}

Kevin M. Bonney* ${ }^{* \dagger}$ and David M. Engman ${ }^{* \dagger}$

From the Departments of Pathology* and Microbiology-Immunology ${ }^{\dagger}$ and the Feinberg Cardiovascular Research Institute, ${ }^{\ddagger}$ Northwestern University, Chicago, Illinois

\author{
Accepted for publication \\ December 23, 2014. \\ Address correspondence to \\ David M. Engman, M.D., \\ Ph.D., Department of Pathol- \\ ogy, Northwestern University, \\ 303 E Chicago Ave, Chicago, \\ IL 60611. E-mail: d-engman@ \\ northwestern.edu.
}

\begin{abstract}
Chagas heart disease is an inflammatory cardiomyopathy that develops in approximately one-third of individuals infected with the protozoan parasite Trypanosoma cruzi. Since the discovery of $T$. cruzi by Carlos Chagas $>100$ years ago, much has been learned about Chagas disease pathogenesis; however, the outcome of $T$. cruzi infection is highly variable and difficult to predict. Many mechanisms have been proposed to promote tissue inflammation, but the determinants and the relative importance of each have yet to be fully elucidated. The notion that some factor other than the parasite significantly contributes to the development of myocarditis was hypothesized by the first physician-scientists who noted the conspicuous absence of parasites in the hearts of those who succumbed to Chagas disease. One of these factors-autoimmunity - has been extensively studied for more than half a century. Although questions regarding the functional role of autoimmunity in the pathogenesis of Chagas disease remain unanswered, the development of autoimmune responses during infection clearly occurs in some individuals, and the implications that this autoimmunity may be pathogenic are significant. In this review, we summarize what is known about the pathogenesis of Chagas heart disease and conclude with a view of the future of Chagas disease diagnosis, pathogenesis, therapy, and prevention, emphasizing recent advances in these areas that aid in the management of Chagas disease. (Am J Pathol 2015, 185: 1537-1547; http://dx.doi.org/10.1016/j.ajpath.2014.12.023)
\end{abstract}

Chagas disease, caused by infection with the protozoan parasite Trypanosoma cruzi, is a neglected tropical disease that affects up to 10 million people worldwide. The disease is endemic in vast areas of the western hemisphere from the United States to Argentina, and many thousands of $T$. cruzi-infected individuals also reside in Canada, Europe, Japan, and Australia. ${ }^{1}$ Although most infected individuals will experience a lifelong subclinical infection, approximately one-third will develop a potentially fatal inflammatory cardiomyopathy and/or megadisease of the gastrointestinal tract. Since the discovery of the illness and its causative agent by Chagas in $1909,{ }^{2}$ there has been extensive basic, translational, and clinical research on this important disease, which has led to the development of several successful strategies for public health intervention and informed approaches to vaccine development and drug discovery. Among the features of this disease, perhaps the most interesting is also the most understated-the tremendous variability in the outcome of infection, largely determined by genetic heterogeneity in both the virulence of specific $T$. cruzi strains and the susceptibility of a specific individual to infection. This unpredictable variability, compounded by differential effects of the physiological state of both parasite and host on the

Supported by NIH grants AI38022 R01-HL075822 and R01-HL80692 (D.M.E.) and American Heart Association Predoctoral Fellowship 0810179Z (K.M.B.).

Disclosures: None declared.

This article is part of a review series on infectious disease.

Current address of K.M.B., Liberal Studies, Faculty of Arts and Science, New York University, New York, NY. 


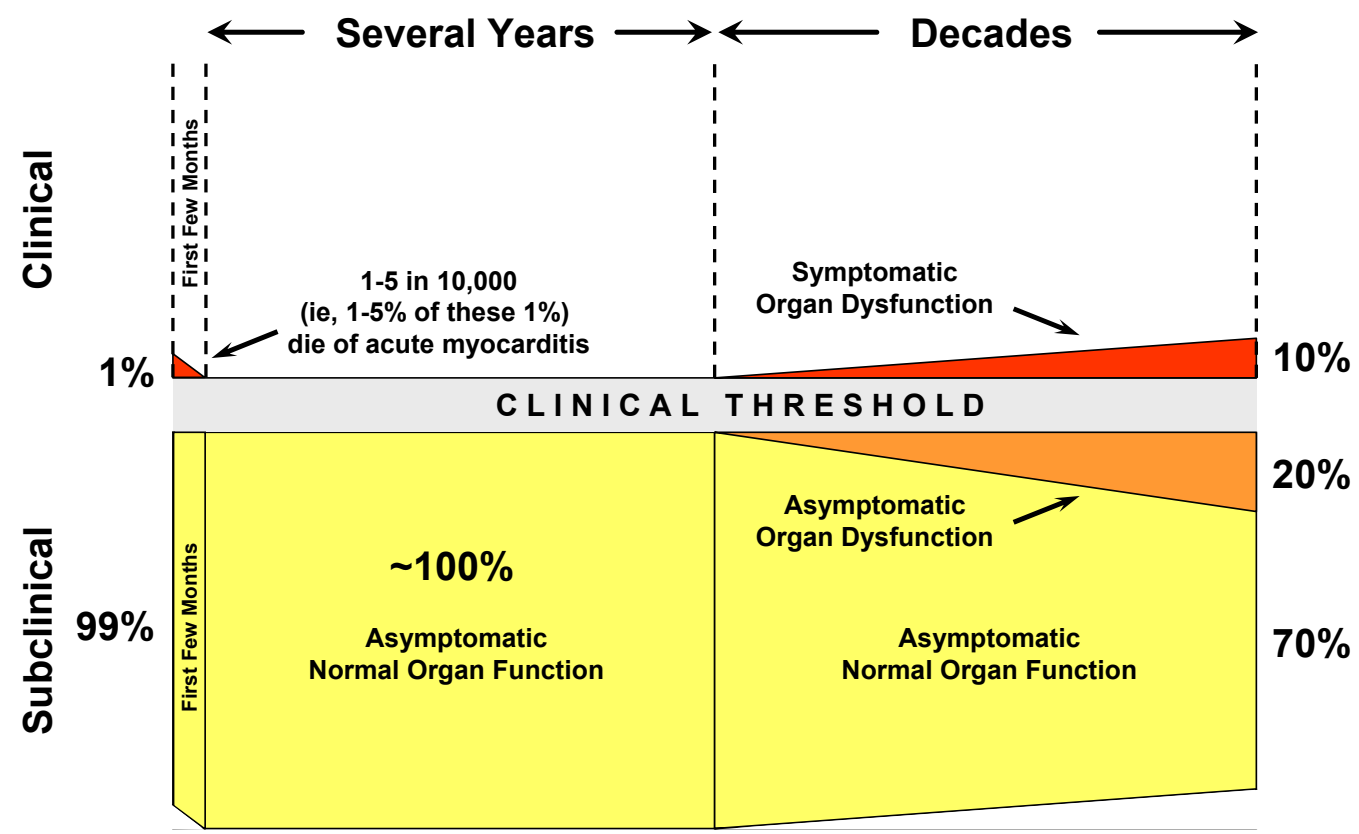

Figure 1 Clinical course of Trypanosoma cruzi infection. During the acute phase of infection, nearly all individuals are unaware of infection, having no signs or symptoms of infection; mild and/or non-specific signs and symptoms, such as anorexia, fever, fatigue, or lymphadenopathy; or more specific, if not pathognomonic, signs of recent infection, such as unilateral periorbital edema (Romaña sign). A few individuals (approximately $1 \%$ ), usually children in endemic regions, develop clinically apparent myocarditis, which normally resolves within several weeks; a few of these children $(0.01 \%$ to $0.05 \%)$ succumb to myocarditis, typically due to fatal dysrhythmias. After this acute phase of a few months' duration, virtually all infected individuals harbor subclinical (silent) disease for a variable time (several years). From that point forward, approximately $30 \%$ of infected individuals develop cardiomyopathy or megadisease during the next several decades, with approximately onethird of these (ie, 10\%) with clinically apparent, symptomatic disease above the clinical threshold. Approximately $20 \%$ develop organ dysfunction that is detectable through clinical testing. Most (approximately 70\%) have lifelong infection with no organ dysfunction. This figure was adapted from Rosenbaum, ${ }^{3}$ copyright 1964 , with permission from Elsevier. The percentages given in this figure are rough estimates, and the outcome of infection may vary greatly depending on variables.

outcome of infection, has complicated efforts to treat and cure Chagas disease for decades.

Trypanosoma cruzi infection is often characterized as having distinct acute, indeterminate, and chronic phases. Although this convention is widely used, we believe that it is easier to understand the course of infection as conveyed by our modification of a scheme by Rosenbaum (Figure 1). ${ }^{3}$ After infection through contact with trypanosome-containing excreta from the reduviid bug vector that transmits $T$. cruzi, consumption of food or beverages contaminated with excreta, or transfusion/transplantation of blood/tissue from an infected donor, the parasite goes through several rounds of host cell invasion, division, and cell lysis for up to several weeks. During this time, parasites can often be detected in the blood. However, most infected individuals demonstrate little, if any, indication of being infected. These are included in the 99\% subclinical group (Figure 1), even though some may develop non-specific symptoms, including anorexia, chills, diarrhea, drowsiness, fever, lethargy, lymphadenopathy, and swelling near the infection site. ${ }^{4}$ Approximately $1 \%$ of the infected individuals, mostly children, develop clinically apparent myocarditis; most recover, but a few (1\% to 5\%) develop fatal complications, including acute heart failure, fatal dysrhythmias, or fulminating meningoencephalitis. ${ }^{4}$ Morbidity and mortality during acute infection can be much higher in some cases. In two microepidemics in northeastern Brazil caused by consumption of $T$. cruzicontaminated food, 12 of 13 infected individuals developed fever and dyspnea, 5 developed congestive heart failure, and 2 died. $^{5}$

In most immunocompetent individuals, parasite-specific adaptive immunity is generally sufficient to keep the parasite at low or undetectable levels. This is the indeterminate phase of Chagas disease. Beginning a variable time after infection (Figure 1), and extending for decades, approximately 30\% will develop chronic Chagas disease, which primarily affects the heart and gastrointestinal tract, but may also involve the nervous and endocrine systems. ${ }^{6,7}$ Approximately one-third of these individuals will have clinically overt cardiomyopathy, typically manifested as congestive heart failure, whereas two-thirds will develop subclinical Chagas disease that is detectable via several diagnostic modalities. Death from chronic Chagas disease is often the result of congestive heart failure after gradual development of myocardial dysfunction due to damage caused by chronic inflammation. ${ }^{6,7}$

In chronic Chagas heart disease (CHD), cardiac myocytes undergo necrosis and cytolysis via various mechanisms, and areas of myocellular hypertrophy and mononuclear cell infiltration develop in the cardiac tissue, with fewer macrophages, eosinophils, neutrophils, and mast cells. ${ }^{4,8-10}$ Fibrosis develops in response to myocardial damage. This remodeling 
may disrupt the cardiac conduction system, leading to dysrhythmias as well as myocardial thinning and cardiac hypertrophy. ${ }^{4}$ Although parasite DNA or antigen can be detected in some inflammatory lesions, intact parasites are frequently absent from the heart in chronic CHD. ${ }^{11}$ In addition to the histopathological changes mentioned earlier in this paragraph, development of occlusive thrombi, apical aneurysm of the dilated left ventricle, decreased contractility of muscle fibers, and destruction of the cardiac conduction system are also commonly found. The associated muscle damage leads to dilation and cardiac dysrhythmias, high-degree heart block, and, ultimately, congestive heart failure, which is the leading cause of death in chronic CHD patients. ${ }^{12}$

Diagnosis of acute $T$. cruzi infection may be made by microscopic analysis of whole blood or buffy coat for the presence of motile bloodform trypomastigotes released from host cells after initial rounds of replication. In chronic Chagas patients, enzyme-linked immunosorbent assay, indirect hemagglutination, or indirect immunofluorescence can be used to test for the presence of $T$. cruzi-specific IgG in sera. ${ }^{4}$ Large-scale screening of the blood supply is underway in many parts of the western hemisphere, with a variety of commercial/industrial immunoassays used; however, several areas with at-risk populations still do not routinely screen all blood products. ${ }^{13}$

To date, only two anti-T. cruzi drug treatments are available, nifurtimox and benznidazole, both of which oxidize the parasite's nucleotide pool, leading to lethal double-stranded DNA breaks. Proper administration of either of these drugs can successfully ameliorate the symptoms of Chagas disease in many individuals, especially those experiencing the acute form of the disease. However, existing treatments for Chagas disease have limited efficacy in certain populations because they require repeated administration over a prolonged time period, and often produce severe adverse effects that cannot be tolerated by all individuals. These characteristics likely suppress compliance with treatment, and subsequently delay resolution of this substantial public health burden. Although the treatment of chronically infected individuals is somewhat controversial, given that most will never develop organ dysfunction, the Latin American Network for Chagas Disease now recommends treatment for all chronically infected individuals. ${ }^{14}$ Heart transplantation may also be performed on patients with severe chronic CHD experiencing congestive heart failure; however, the reappearance of $T$. cruzi infection may occur with immunosuppressive therapy after the transplantation procedure. Currently, no effective vaccine is available for preventing $T$. cruzi infection, although many share the view that the outlook is more promising now than in the past. ${ }^{15}$

\section{Mechanisms of Disease Pathogenesis}

Several distinct mechanisms have been proposed to account for the widely varying cardiac pathology observed in
T. cruzi-infected individuals. These mechanisms include damage to cardiomyocytes that is directly mediated by live parasites, bystander damage resulting from exposure to parasite-specific immune responses, cellular damage due to the effects of non-specific immune responses in the local microenvironment, microvasculopathy leading to ischemia and inflammation, and autoimmunity, which may result from several pathogenic mechanisms (Figure 2). Most published research on CHD pathogenesis addresses two issues-parasite persistence and autoimmunity. This does not mean that the other mechanisms are not operative or important - just that they have not been as well investigated.

In any individual who develops disease, one or more of these mechanisms may contribute to pathogenesis. This issue is further complicated by the fact that infections commonly involve mixtures of parasite clones, each having unique pathogenic potential (ie, polyparasitism). ${ }^{16}$ Trypanosoma cruzi clonal variation may influence tissue tropism and the ability to establish a chronic infection, whereas host genetic polymorphisms that affect cytokine production may modulate disease severity and even the mechanism of pathogenesis. ${ }^{17-21}$ Integration of T. cruzi kinetoplast DNA into the genomic DNA of host cells may further alter the outcome of infection. ${ }^{6,22,23}$ Determining the contributions of one mechanism or another is also extremely difficult in the presence of live T. cruzi infection, in which multiple coincident mechanisms may be operative. However, the current body of evidence suggests that parasite is a key requirement for every mechanism.

\section{Parasite Persistence}

An obvious explanation for the myocarditis associated with T. cruzi infection was based on reports of parasites in the inflamed hearts of deceased Chagas patients and the presumption that the chronic inflammation and myocardial fibrosis associated with $T$. cruzi infection were primarily the result of a reparative process initiated to ameliorate the damage to myocytes that was mechanically inflicted by live parasites. ${ }^{6}$ A direct correlation between parasite persistence and inflammation has been proposed on the basis of detection of $T$. cruzi antigens and genetic markers via PCR in heart sections showing signs of myocarditis. ${ }^{24,25}$

Lysis of host cells by $T$. cruzi is undoubtedly responsible for some degree of cardiac tissue damage (Figure 2A). Robust cardiac infiltration by leukocytes may also cause mechanical disruption to the myocardium and associated vasculature and innervation (Figure 2B), and inflammatory mediators released by lymphocytes, macrophages, eosinophils, neutrophils, and mast cells may cause further bystander damage to myocytes (Figure 2C). ${ }^{4,26}$ Trypanosoma cruzi is capable of subverting the host bradykinin system by activating bradykinin B2 receptors during endothelial cell invasion, leading to vasodilation and subsequent interstitial edema. ${ }^{27}$ Trypanosoma cruzi also produces several bioactive lipids, including thromboxane $\mathrm{A}$ and 
A

Direct Damage by Parasite

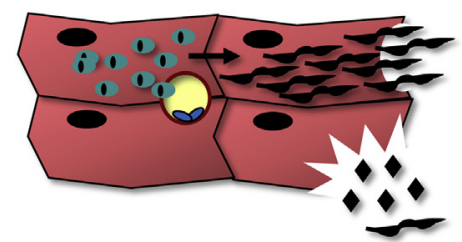

C



E
B


D

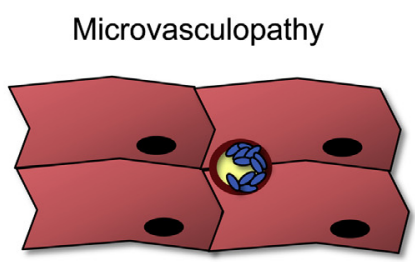

Autoimmunity

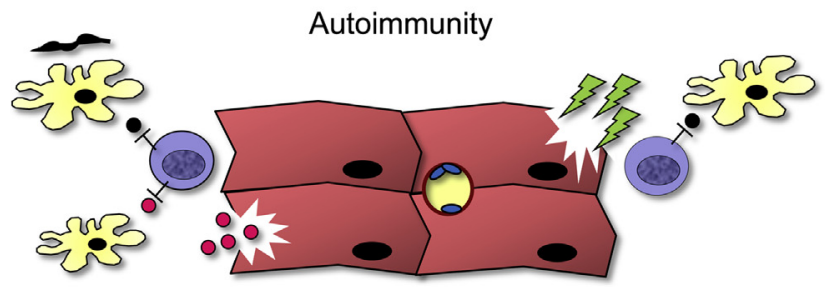



( Platelet

Blood vessel

Figure 2 Mechanisms by which T. cruzi infection can cause myocarditis. A: Direct damage by parasites caused by cell lysis by trypomastigotes that have differentiated from intracellular amastigotes (top panels) or a likely toxic (lytic) product of trypomastigotes (bottom panels). B: Parasite-specific immunity may contribute to cardiac pathology due to the destruction and displacement of myocytes, followed by mononuclear infiltration and fibrosis. C: Non-specific damage caused by innate immune responses, granulocyte activation, and antibody-mediated cytotoxicity may cause bystander injury to cardiomyocytes. D: Microvasculopathy leading to ischemia, including occlusive platelet aggregation. E: Parasite-induced autoimmunity generated by molecular mimicry between parasite and self-antigens (left panels) or bystander activation of autoreactive T cells after cell lysis by $T$. cruzi (right panels).

prostaglandin $\mathrm{F} 2 \alpha$, which promote vascular constriction, platelet aggregation, and vascular smooth muscle proliferation (Figure 2D) ${ }^{28}$ Another mechanism through which live parasites may induce myocardial damage is via the induction of oxidative stress-induced injury. It has been shown that oxidative damage to mitochondrial respiratory complexes in T. cruzi-infected cells leads to reduced mitochondrial ATP generation and deficiencies in cellular antioxidant defense mechanisms, such as reduced levels of superoxide dismutase,$^{29}$ and the level of oxidative injury biomarkers in the myocardium positively correlates with chronic disease severity. ${ }^{29}$ Most recently, it was determined that cardiac miRNAs are dysregulated during T. cruzi infection, and several of these regulate key signaling pathways that are likely involved in disease pathogenesis. ${ }^{30}$

Others have hypothesized that production of some type of toxin may contribute to parasite-mediated damage (Figure 2A). Although no conventional toxins have been identified in $T$. cruzi, the parasite produces some substances that may have substantial toxic effects on cells in vivo. Two examples of such substances are TC-Tox and LYT1, both acid-active hemolysins presumably involved in escape of the parasite from the parasitophorous vacuole into the cytoplasm of infected cells. ${ }^{31,32}$ Interestingly, both of these proteins immunologically cross-react with human complement protein $\mathrm{C} 9$, although considerable dissimilarity exists between them on both the DNA sequence and protein level. It is unlikely that these, or similar molecules if they exist, play a significant role in CHD pathogenesis, but it is conceivable that some amount of tissue injury may occur secondary to cellular damage inflicted by these pore-forming proteins.

Although dogma presumes that most infiltrating lymphocytes recognize specific parasite antigens, this has not been definitely proved. Notably, it has been widely documented that the degree of tissue parasitism does not correlate with the severity of Chagas cardiomyopathy, and even the application of highly sensitive techniques, such as PCR and immunohistochemistry, has variably found correlation ${ }^{24}$ or lack of correlation $^{27,33}$ between the presence of parasite antigen/ DNA and tissue inflammation. In an extensive, highly detailed histopathological analysis of CHD, Laranja et al ${ }^{33}$ found that parasites were rarely present in $>20 \%$ of tissue sections containing significant inflammation. These findings, in conjunction with the extant evidence that other mechanisms, such as autoimmunity, are operative in $T$. cruziinfected humans and experimental animals, indicate that the pathogenic immune response generated in individuals with CHD is not entirely parasite specific. Indeed, the variability in cardiac histopathology in CHD likely reflects the heterogeneity in the inflammatory mechanism involved. Different 

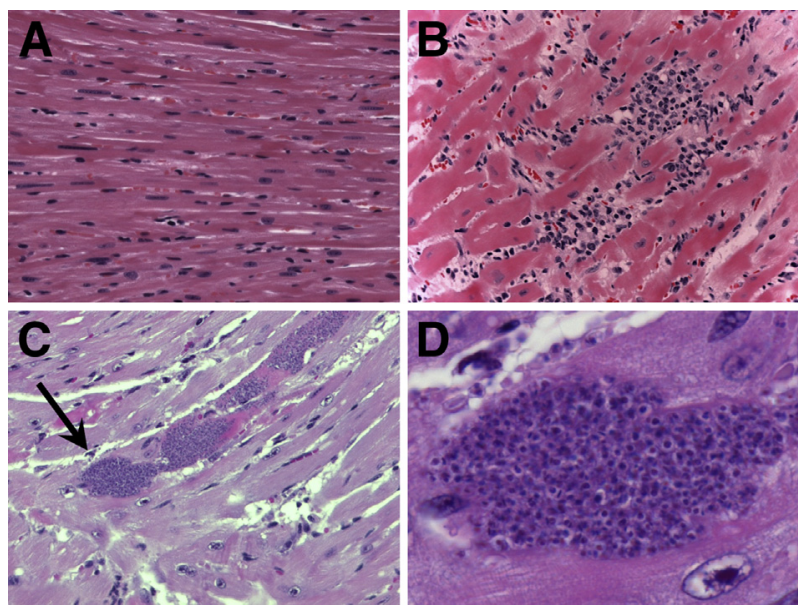

Figure 3 Different histological outcomes of Trypanosoma cruzi infection in the heart. The cardiac pathological manifestations of infection range from no inflammation to focal carditis to pancarditis, each of which can develop with or without focal, regional, or massive tissue parasitosis. The complex interplay between parasite pathogenicity, host susceptibility, and physiological variables determines disease outcome. Shown here are four representative images of hematoxylin and eosin-stained cardiac sections. A: Normal histology, which can occur with a low virulence T. cruzi strain or a resistant host. B: Mononuclear cell infiltration, fibrosis, and edema, without parasites, which can occur during infection with certain $T$. cruzi strains and/or human or animal hosts. C: Inflammation with parasitosis. D: Higher magnification of the parasite pseudocyst marked by the arrow in C. The pseudocyst is filled with intracellular amastigotes. Original magnification: $\times 40($ A-C); $\times 160$ (D).

combinations of parasite and host strains can be used for experimental infections having three basic cardiac histological outcomes: normal histology (Figure 3A), inflammation without parasitosis (Figure 3B), and inflammation with parasitosis (Figure 3, C and D). The degree of inflammation can vary from extremely mild to extremely severe (Figure 3 , B and C).

\section{Autoimmunity}

The autoimmunity hypothesis suggests that cardiac damage, regardless of initial cause, leads to a breakdown of self-tolerance, resulting in an immune reaction against selfproteins. Cardiac autoimmunity may be initiated by parasite-induced damage to cardiomyocytes ${ }^{34,35}$ and/or by molecular mimicry between immunologically similar epitopes of parasite and host proteins (Figure 2E) ${ }^{36-38}$ During bystander activation, mechanical damage caused directly by infective $T$. cruzi, followed by subsequent parasite-specific and non-specific immune responses, would result in the release of copious amounts of selfantigens in an environment particularly rich in inflammatory mediators, including cytokines and chemokines, lymphotoxin, nitric oxide, and granule components from eosinophils and polymorphonuclear cells. ${ }^{39}$ This milieu of potent immune stimuli may overcome the threshold of activation needed to breach self-tolerance, and stimulate cytotoxic $\mathrm{T}$ cells without directly triggering the $\mathrm{T}$-cell receptor, resulting in autoimmunity targeted against multiple antigens. Bystander activation involving $\mathrm{CD}^{+} \mathrm{T}$ cells may also occur in response to $T$. cruzi antigens that are presented on the major histocompatibility complex I molecules of infected cells. Inflammatory factors present in the local environment, such as interferon- $\gamma$ and nitric oxide, are known to promote the activation of potentially autoreactive $\mathrm{T}$ cells encountering major histocompatibility complex-bound cognate antigen. Once activated, autoreactive lymphocytes may proliferate in response to selfantigen presented on host antigen-presenting cells. Molecular mimicry between parasite and host antigens may also contribute to Chagas pathogenesis by driving a deleterious autoimmune response, as has been demonstrated for experimental autoimmune encephalomyelitis (EAE), a better understood model of autoimmune pathology induced by molecular mimicry that is a surrogate for studying aspects of multiple sclerosis pathogenesis in humans. ${ }^{40}$ The potential to extend the knowledge of autoimmunity in Chagas disease to understand other infectious diseases with autoimmune components and to develop potential treatments further supports continued study of $T$. cruzi-induced autoimmunity.

\section{History and Future of Autoimmunity in CHD}

The earliest discussions of autoimmunity in the literature did not use this term, but rather refer to allergic or hyperergic mechanisms of pathogenesis, perhaps induced by infection but ultimately independent of the parasite. ${ }^{41}$ This view was reinforced by the finding that cardiac autoantibodies could induce myocarditis in rats ${ }^{42}$ and the establishment of the first experimental autoimmune myocarditis models. ${ }^{43}$ Autoimmunity has been considered an important contributor to the complex immune response that develops in $T$. cruzi-infected individuals since the mid-1970s, when it was observed by Santos-Buch and Teixeira ${ }^{44}$ that $T$. cruzi infection promoted rejection of allogeneic heart cell transplants, and that lymphocytes from T. cruzi-infected animals rapidly destroyed embryonic cardiomyocytes in culture. This work, together with those of Cossio et $\mathrm{al}^{45}$ on the endocardial-vascular-interstitial antibody and of Ribeiro dos Santos et $\mathrm{al}^{46}$ on immunoglobulin and complement deposition on neurons, was highlighted in a commentary in the British Medical Journal in $1977 .^{47}$

By the late 1970s, some prominent researchers were cautioning those working on the development of $T$. cruzispecific vaccines and other immunomodulatory therapies because of the poorly gauged possibility of inadvertently inducing potentially fatal autoimmunity. ${ }^{48}$ However, reports that protective $T$. cruzi-specific immunity could be induced without eliciting pathogenic autoimmunity were soon published, providing some assurance that parasite-specific therapeutics could be used without the concern that exposure to $T$. cruzi antigens in the presence of immune-activating 
adjuvants might induce harmful autoreactive responses. ${ }^{49,50}$ The debate about a role for autoimmunity in Chagas disease, and the quest for clues to the etiology of this phenomenon, continued to unfold in a series of articles published in the 1980s. ${ }^{51-54}$ Autoreactive T cells and antibodies were identified in individuals with chronic Chagas disease, and several specific antigens abundant in the myocardium were identified as targets of autoreactive responses. ${ }^{53-57}$

By the 1990s, it had been proposed by several groups that molecular mimicry between $T$. cruzi antigens and host antigens was involved in the induction of autoimmunity. ${ }^{58-60}$ Several T. cruzi proteins, including ribosomal $\mathrm{P}$ protein and B13, were identified as cross-reactive to host proteins, such as cardiac myosin, ${ }^{61-63}$ lymphocyte antigens, ${ }^{64}$ neuronal tisues, ${ }^{65}$ human ribosomal $\mathrm{P}$ proteins, ${ }^{36,66}$ muscle antigens, ${ }^{67}$ and small nuclear ribonucleoprotein. ${ }^{68}$ Antibodies targeting $\beta 1$ - and $\beta 2$-adrenergic receptors in human Chagas patients were then linked to the development of atrial arrythmias and dilated cardiomyopathy, ${ }^{69}$ and antibodies specific for human $\mathrm{m} 2$ muscarinic acetylcholine receptors were identified in patients with Chagas disease as a potential contributor to Chagas' cardioneuromyopathy. ${ }^{70}$ Antibodydependent cytotoxicity, whereby antibodies present in the sera of chronically $T$. cruzi-infected mice bind to and mediate destruction of myocytes, was identified as another potential mechanism of autoimmune pathology. ${ }^{71}$

A series of reports describing robust autoreactive T-cell and antibody responses specific for cardiac myosin, combined with findings that molecular mimicry and breach of immunological tolerance to cardiac autoantigens trigger potentially pathogenic autoimmunity, were published in the early 2000s. ${ }^{72-75}$ Potential targets for cross-reactive antibodies in sera from Chagas patients and experimentally infected animals were identified as ribosomal proteins, G-protein-coupled receptors, neuronal proteins, and at least one novel host protein named Cha. ${ }^{46,57,62,63,76-78}$ Interestingly, immunization of mice with the $T$. cruzi cysteine protease cruzipain induced autoantibodies that pathologically inhibit the function of cardiac muscarinic acetylcholine receptors, whereas a DNA vaccine containing the genes that encode several T. cruzi proteins, including cruzipain, was later found to provide protective immunity against $T$. cruzi infection without causing cardiac abnormalities. ${ }^{79}$ The ability of cruzipain to modulate antigen expression by macrophages and induce a proinflammatory cytokine response likely contributes to both scenarios. $^{80}$

Exposure to $T$. cruzi antigens in the absence of infection with live parasites was shown to be sufficient to induce both autoimmunity and a modest, but detectable, level of cardiomyocyte damage, as evidenced by production of polyantigenic humoral and cell-mediated autoimmunity and release of cardiac troponin I into the serum, respectively. $^{73,81}$ The idea behind the latter finding was that the autoimmune response to $T$. cruzi infection would exhibit epitope spreading, ${ }^{82}$ the stepwise temporal development of peptide-specific autoimmune responses, as it does in mouse models of multiple sclerosis. ${ }^{83}$ Rather, T. cruzi infection leads to an explosion of autoimmune responses to many cardiac antigens simultaneously. ${ }^{82} \mathrm{We}$ found that treatment of $T$. cruzi-infected mice with benznidazole to eliminate the parasite also resulted in a resolution of cardiac pathology and humoral and cell-mediated autoimmunity. ${ }^{34}$ Reinfection caused reestablishment of both autoimmunity and cardiac inflammation, providing the first clear link between parasite persistence and autoimmunity that could potentially reconcile the two camps.

However, subsequent reviews and reports resumed the ongoing debate, with some arguing that autoimmunity is an epiphenomenon of $T$. cruzi infection in some cases, and others maintaining that an autoimmune mechanism of pathogenesis has not been adequately tested. ${ }^{13,23,81,84-87}$ Although the data describing the existence of $T$. cruziinduced autoimmunity and suggesting links to Chagas pathogenesis continue to grow, those who dismiss the significance seem confident that it is irrelevant to disease pathogenesis in the absence of direct reported evidence. Ongoing efforts to develop and administer T. cruzi antigen- and DNAbased vaccines will soon help to address whether protective immunity against $T$. cruzi can be achieved in large populations with no adverse health effects due to autoimmunity. In the case of at least one antigen-based vaccine, which targets a $T$. cruzi $24 \mathrm{kDa}$ trypomastigote excretory-secretory protein (Tc24) and $T$. cruzi trypomastigote surface transialidase (TSA-1), preliminary data have been promising and will likely lead to the start of clinical trials within the next few years. ${ }^{88}$ With other groups simultaneously working on several other antigen and DNA vaccine candidates, continued study of Chagas disease autoimmunity is important. Elucidating the mechanism of Chagas autoimmunity can help address potential complications of the treatments involving autoimmunity, and may serve as a model for studying infection-induced autoimmunity to gain insights, which may apply to other diseases. ${ }^{79,88-90}$

\section{Perspective}

Despite extensive research, the ability to reliably predict and prevent disease outcome in $T$. cruzi-infected individuals remains elusive. Of the factors that contribute to the notable heterogeneity and complexity of Chagas disease, autoimmunity, and its unclear role in the pathogenesis of CHD, is one of the most interesting factors. Clearly, in some humans, autoantibodies and autoreactive $\mathrm{T}$ cells are produced during T. cruzi infection, with the autoantibodies having proven chronotropic effects on cardiac function. In experimental animals, many detailed studies have described humoral and cellular autoimmune responses, identified cardiac autoantigens and parasite mimic antigens, demonstrated the pathogenic potential of anti-receptor autoantibodies, and shown that autoimmune-mediated cardiac tissue rejection can occur. 
The greatest challenge is to determine the significance of a potentially pathogenic mechanism, when multiple coincident mechanisms of tissue inflammation are likely. Of particular importance are the questions of how often autoimmunity develops in $T$. cruzi-infected individuals and whether the autoimmunity leads to cardiac inflammation. Cardiac myosin heavy chain is a particularly interesting target of humoral and cellular autoimmunity associated with $T$. cruzi infection, because it has been implicated in other cardiac infections, including chlamydial and coxsackieviral myocarditis. Because myosin autoimmunity may be pathogenic, we inhibited the myosin autoimmune response in A/J mice infected with the Brazil strain of $T$. cruzi using myosin-specific peripheral tolerance induction. ${ }^{91}$ Although both myosin- and T. cruzi-specific immunity were significantly decreased (the latter because of molecular mimicry), the mice still developed myocarditis, suggesting the involvement of other potential cardiac autoantigens and excluding autoimmunity. It is most challenging to address this in an antigen-specific manner because there are so many potential autoantigens involved. Further research needs to be conducted to substantiate the importance of autoimmunity in CHD pathogenesis.

We were initially surprised that immunization with $T$. cruzi lysate ${ }^{73}$ or heat-killed T. cruzi $^{92}$ did not induce histologically evident cardiac inflammation despite inducing cardiac myosin autoimmunity of a magnitude similar to that resulting from immunization with purified cardiac myosin. What are the factors that change an autoimmune response from nonpathogenic to pathogenic? This exact question has been addressed in virus-induced EAE. ${ }^{40}$ Immunization of SJL/J mice with the major encephalitogenic myelin peptide PLP $_{139-151}$ in complete Freund adjuvant induces EAE, whereas immunization with a Haemophilus influenzae peptide sharing 6 of 13 amino acids with PLP $139-151$ (HI mimic) in complete Freund adjuvant does not. However, when mice were infected with a nonpathogenic Theiler murine encephalomyelitis virus engineered to express $\mathrm{PLP}_{139-151}$ or the HI mimic, all of the mice developed EAE. It is believed that the virus provides additional innate immune signals that convert nonpathogenic autoimmune responses to pathogenic ones. This possibility is testable in the context of experimental $T$. cruzi infection. Furthermore, the study of either disease may have implications for the investigation and development of treatments for other infectioninduced, organ-specific diseases, such as multiple sclerosis and viral myocarditis, that may have an autoimmune component.

More important, it seems clear that the parasite is essential, for both initiating autoimmunity and maintaining it over time. ${ }^{34}$ Although parasite persistence and parasitespecific immunity are clearly involved in pathogenesis in many cases, and parasite antigen and DNA may be detected in parasite-free areas of the heart, there are clearly situations in which there is inflammation in the absence of $T$. cruzi parasites or molecules, as noted by generations of pathologists. The recent development of highly luminescent parasites and the finding of chronic myocarditis in the absence of T. cruzi further support this notion. ${ }^{93}$

Finally, the mere presence of parasites in an inflammatory lesion does not preclude the presence of a coincident autoimmune response in the same lesion. Most authors conclude that Chagas is a complex disease with many mechanisms, which is an understatement at best. The individual mechanism in the setting of live infection can be dissected by inhibiting the individual mechanism; however, such an intervention often affects more than one potential mechanism. For example, interventions to reduce autoimmunity, such as peripheral tolerance induction, can inadvertently suppress protective parasite-specific immune responses. There are experimental models of individual mechanisms, such as autoimmunity, vaso-occlusion, and mitochondrial dysfunction. Although these models may recapitulate the aspects of pathogenesis in isolation, they may only be artificially robust and not adequately reflect the physiological aspects of infection. One such example is the induction of myocarditis via adoptive transfer of lymphocytes. However, despite a controlled setting, ex vivo stimulation with self-antigen or mitogen does not confirm that the same cells are inflammatory in the physiological setting.

Although the transmission of $T$. cruzi has significantly decreased in recent years due to improved vector control, T. cruzi-induced CHD remains a major health problem in vast areas of the western hemisphere. Much knowledge about the epidemiology, clinical disease progression, and pathogenesis of CHD has been gained during the past century, but there are many aspects of the disease that remain unclear and warrant further investigation. The study of a wide variety of animal models with different strain-strain combinations of parasite and host has allowed for the investigation of specific aspects of the disease that might manifest themselves specifically in infected individuals. From these studies, it is likely that both parasitemediated damage and parasite-specific immunity and autoimmunity contribute to disease progression. Also, rather than being mutually exclusive, it is likely that these mechanisms act coincidentally or even synergistically (eg, by generating a proinflammatory environment that promotes both parasite killing and autoimmunity) to promote disease progression.

Further investigation into the potential interactions among the various mechanisms of Chagas pathogenesis described herein will allow us to develop safer, more efficient therapies and perhaps even cures for T. cruzi infection and Chagas disease. There have been some promising recent developments in the therapeutic area, including testing of cell-based therapies involving transfer of bone marrow cells or mesenchymal stem cells. ${ }^{94-96}$ This is an exciting avenue that has potential to slow or reverse cardiac dysfunction while maintaining robust parasite-specific immunity. A serious clinical trial investigating the incidence, quality, and 
magnitude of humoral and cellular cardiac autoimmunity in infected individuals is needed to fully understand the potentially important clinical problem of $T$. cruzi-induced autoimmunity. Although not specifically focused on autoimmunity, recent genome-wide association studies and specific investigations of polymorphisms in cytokine genes $^{97,98}$ may someday reveal the genetic basis for susceptibility and resistance in humans.

Although benznidazole and nifurtimox remain the mainstay of treatment of $T$. cruzi infection, there have been some recent developments in drug discovery, ${ }^{99}$ and we are all hopeful that redoubled efforts in this area will lead to new individual or combination therapies to cure infection without the toxicity of the current agents. The determination of the $T$. cruzi genome sequence and comparative genomics of various $T$. cruzi strains should also reveal parasitespecific biosynthetic pathways revealing new targets for drug discovery. Vaccine development has enjoyed many recent advances, particularly because it is clear that reduction or elimination of the parasite load has great therapeutic benefit. $^{79,88,90,100-102}$ Development of an effective $T$. cruzi $^{-}$ vaccine may one day preclude the need for improving existing therapies. However, until an effective vaccine or improved therapies are widely available, global efforts to treat and prevent Chagas disease must also emphasize increased disease surveillance, including screening of potentially contaminated blood, organ, and food supplies in areas where infection is emerging. Vector control is also an area that deserves ongoing attention and support.

\section{Conclusions}

Chagas disease remains a major public health problem throughout the Americas, where transmission via transfusion/transplantation and through contact with infected insects continues to be a matter of concern. The tremendous variability in the outcome of infection and the multiple known mechanisms of tissue inflammation pose significant challenges to those trying to understand disease pathogenesis and work toward new therapies and vaccines.

\section{Acknowledgments}

We thank Randal Tibbetts, Lisa Godsel, Juan Leon, Kegiang Wang, Kenneth Hyland, Melvin Daniels, Joann Taylor, Conrad Epting, and Stephen Miller for their contributions to our research on Chagas heart disease autoimmunity during the past decade.

\section{References}

1. Bonney KM: Chagas disease in the 21st century: a public health success or an emerging threat? Parasite 2014, 21:11

2. Chagas C: Nova trypanosomíase humana: estudo sobre a morfologia e o ciclo evolutivo do Schizotripanum cruzi n. gen. Sp, ajente etiolójico de nova entidade mórbida do homem. Mem Inst Oswaldo Cruz 1909, 1:159-218

3. Rosenbaum MB: Chagasic myocardiopathy. Prog Cardiovasc Dis 1964, 7:199-225

4. Tanowitz HB, Kirchhoff LV, Simon D, Morris SA, Weiss LM, Wittner M: Chagas' disease. Clin Microbiol Rev 1992, 5: 400-419

5. Bastos CJ, Aras R, Mota G, Reis F, Dias JP, de Jesus RS, Freire MS, de Araujo EG, Prazeres J, Grassi MF: Clinical outcomes of thirteen patients with acute Chagas disease acquired through oral transmission from two urban outbreaks in northeastern Brazil. PLoS Negl Trop Dis 2010, 4:e711

6. Teixeira AR, Nascimento RJ, Sturm NR: Evolution and pathology in Chagas disease: a review. Mem Inst Oswaldo Cruz 2006, 101: 463-491

7. Andrade ZA: Immunopathology of Chagas disease. Mem Inst Oswaldo Cruz 1999, 94(Suppl 1):71-80

8. Melo RC, D'Avila H, Fabrino DL, Almeida PE, Bozza PT: Macrophage lipid body induction by Chagas disease in vivo: putative intracellular domains for eicosanoid formation during infection. Tissue Cell 2003, 35:59-67

9. Elias FE, Vigliano CA, Laguens RP, Levin MJ, Berek C: Analysis of the presence of Trypanosoma cruzi in the heart tissue of three patients with chronic Chagas' heart disease. Am J Trop Med Hyg 2003, 68 : 242-247

10. Postan M, Correa R, Ferrans VJ, Tarleton RL: In vitro culture of cardiac mast cells from mice experimentally infected with Trypanosoma cruzi. Int Arch Allergy Immunol 1994, 105:251-257

11. Tarleton RL, Zhang L, Downs MO: "Autoimmune rejection" of neonatal heart transplants in experimental Chagas disease is a parasite-specific response to infected host tissue. Proc Natl Acad Sci U S A 1997, 94:3932-3937

12. Rodriguez-Salas LA, Klein E, Acquatella H, Catalioti F, Davalos VV, Gomez-Mancebo JR, Gonzalez H, Bosch F, Puigbo JJ: Echocardiographic and clinical predictors of mortality in chronic Chagas' disease. Echocardiography 1998, 15:271-278

13. Tanowitz HB, Machado FS, Jelicks LA, Shirani J, de Carvalho AC, Spray DC, Factor SM, Kirchhoff LV, Weiss LM: Perspectives on Trypanosoma cruzi-induced heart disease (Chagas disease). Prog Cardiovasc Dis 2009, 51:524-539

14. Viotti R, Alarcón de Noya B, Araujo-Jorge T, Grijalva MJ, Guhl F, López MC, Ramsey JM, Ribeiro I, Schijman AG, Sosa-Estani S, Torrico F, Gascon J; Latin American Network for Chagas Disease, NHEPACHA: Towards a paradigm shift in the treatment of chronic Chagas disease. Antimicrob Agents Chemother 2014, 58:635-639

15. Tarleton RL, Reithinger R, Urbina JA, Kitron U, Gurtler RE: The challenges of Chagas Disease: grim outlook or glimmer of hope. PLoS Med 2007, 4:e332

16. Perez CJ, Lymbery AJ, Thompson RC: Chagas disease: the challenge of polyparasitism? Trends Parasitol 2014, 30:176-182

17. Macedo AM, Oliveira RP, Pena SD: Chagas disease: role of parasite genetic variation in pathogenesis. Expert Rev Mol Med 2002, 4: $1-16$

18. Rodriguez HO, Guerrero NA, Fortes A, Santi-Rocca J, Gironès N, Fresno M: Trypanosoma cruzi strains cause different myocarditis patterns in infected mice. Acta Trop 2014, 139:57-66

19. Costa GC, da Costa Rocha MO, Moreira PR, Menezes CA, Silva MR, Gollob KJ, Dutra WO: Functional IL-10 gene polymorphism is associated with Chagas disease cardiomyopathy. J Infect Dis 2009, 199:451-454

20. Zafra G, Morillo C, Martin J, Gonzalez A, Gonzalez CI: Polymorphism in the 3' UTR of the IL12B gene is associated with Chagas' disease cardiomyopathy. Microbes Infect 2007, 9:1049-1052

21. Calzada JE, Nieto A, Beraun Y, Martin J: Chemokine receptor CCR5 polymorphisms and Chagas' disease cardiomyopathy. Tissue Antigens 2001, 58:154-158

22. Simöes-Barbosa A, Barros AM, Nitz N, Argañaraz ER, Teixeira AR: Integration of Trypanosoma cruzi kDNA minicircle sequence in the 
host genome may be associated with autoimmune serum factors in Chagas disease patients. Mem Inst Oswaldo Cruz 1999, 94(Suppl 1): 249-252

23. Teixeira AR, Hecht MM, Guimaro MC, Sousa AO, Nitz N: Pathogenesis of Chagas disease: parasite persistence and autoimmunity. Clin Microbiol Rev 2011, 24:592-630

24. Bellotti G, Bocchi EA, de Moraes AV, Higuchi ML, BarberoMarcial M, Sosa E, Esteves-Filho A, Kalil R, Weiss R, Jatene A, Pileggi F: In vivo detection of Trypanosoma cruzi antigens in hearts of patients with chronic Chagas' heart disease. Am Heart J 1996, 131: 301-307

25. Zhang L, Tarleton RL: Parasite persistence correlates with disease severity and localization in chronic Chagas' disease. J Infect Dis 1999, 180:480-486

26. Ribeiro Dos Santos R, Hudson L: Trypanosoma cruzi: immunological consequences of parasite modification of host cells. Clin Exp Immunol 1980, 40:36-41

27. Todorov AG, Andrade D, Pesquero JB, Araujo Rde C, Bader M, Stewart J, Gera L, Muller-Esterl W, Morandi V, Goldenberg RC, Neto HC, Scharfstein J: Trypanosoma cruzi induces edematogenic responses in mice and invades cardiomyocytes and endothelial cells in vitro by activating distinct kinin receptor (B1/B2) subtypes. FASEB J 2003, 17:73-75

28. Ashton AW, Mukherjee S, Nagajyothi FN, Huang H, Braunstein VL, Desruisseaux MS, Factor SM, Lopez L, Berman JW, Wittner M, Scherer PE, Capra V, Coffman TM, Serhan CN, Gotlinger K, Wu KK, Weiss LM, Tanowitz HB: Thromboxane A2 is a key regulator of pathogenesis during Trypanosoma cruzi infection. J Exp Med 2007, 204:929-940

29. Wen JJ, Vyatkina G, Garg N: Oxidative damage during chagasic cardiomyopathy development: role of mitochondrial oxidant release and inefficient antioxidant defense. Free Radic Biol Med 2004, 37: $1821-1833$

30. Ferreira LR, Frade AF, Santos RH, Teixeira PC, Baron MA, Navarro IC, Benvenuti LA, Fiorelli AI, Bocchi EA, Stolf NA, Chevillard C, Kalil J, Cunha-Neto E: MicroRNAs miR-1, miR-133a, miR-133b, miR-208a and miR-208b are dysregulated in chronic Chagas disease cardiomyopathy. Int J Cardiol 2014, 175:409-417

31. Andrews NW, Abrams CK, Slatin SL, Griffiths G: A T. cruzisecreted protein immunologically related to the complement component C9: evidence for membrane pore-forming activity at low $\mathrm{pH}$. Cell 1990, 61:1277-1287

32. Manning-Cela R, Cortes A, Gonzalez-Rey E, Van Voorhis WC, Swindle J, Gonzalez A: LYT1 protein is required for efficient in vitro infection by Trypanosoma cruzi. Infect Immun 2001, 69: 3916-3923

33. Laranja FS, Dias E, Nobrego G, Marinda A: Chagas' disease: a clinical, epidemiologic and pathologic study. Circulation 1956, 14: $1035-1060$

34. Hyland KV, Leon JS, Daniels MD, Giafis N, Woods LM, Bahk TJ, Wang K, Engman DM: Modulation of autoimmunity by treatment of an infectious disease. Infect Immun 2007, 75:3641-3650

35. Fujinami RS, von Herrath MG, Christen U, Whitton JL: Molecular mimicry, bystander activation, or viral persistence: infections and autoimmune disease. Clin Microbiol Rev 2006, 19:80-94

36. Kaplan D, Ferrari I, Bergami PL, Mahler E, Levitus G, Chiale P, Hoebeke J, Van Regenmortel MH, Levin MJ: Antibodies to ribosomal P proteins of Trypanosoma cruzi in Chagas disease possess functional autoreactivity with heart tissue and differ from anti-P autoantibodies in lupus. Proc Natl Acad Sci U S A 1997, 94: 10301-10306

37. Motran CC, Fretes RE, Cerban FM, Rivarola HW, Vottero de Cima E: Immunization with the C-terminal region of Trypanosoma cruzi ribosomal P1 and P2 proteins induces long-term duration cross-reactive antibodies with heart functional and structural alterations in young and aged mice. Clin Immunol 2000, 97:89-94
38. Cunha-Neto E, Bilate AM, Hyland KV, Fonseca SG, Kalil J, Engman DM: Induction of cardiac autoimmunity in Chagas heart disease: a case for molecular mimicry. Autoimmunity 2006, 39: $41-54$

39. Engman DM, Leon JS: Pathogenesis of Chagas heart disease: role of autoimmunity. Acta Trop 2002, 81:123-132

40. Croxford JL, Anger HA, Miller SD: Viral delivery of an epitope from Haemophilus influenzae induces central nervous system autoimmune disease by molecular mimicry. J Immunol 2005, 174:907-917

41. Torres C: Patologia de la miocarditis crónica en la enfermedad de Chagas. An 5a Reun Soc Argent Pat Reg Norte 1929, 2: 902-916

42. Cavelti PA: Studies on the pathogenesis of rheumatic fever: cardiac lesions produced in rats by means of autoantibodies to heart and connective tissues. Arch Pathol 1947, 44:13-27

43. Jaffe R, Holz E: Experimental allergic myocarditis. Exp Med Surg 1948, 6:189-202

44. Santos-Buch CA, Teixeira AR: The immunology of experimental Chagas' disease, 3: rejection of allogeneic heart cells in vitro. J Exp Med 1974, 140:38-53

45. Cossio PM, Diez C, Szarfman A, Kreutzer E, Candiolo B, Arana RM: Chagasic cardiopathy: demonstration of a serum gamma globulin factor which reacts with endocardium and vascular structures. Circulation 1974, 49:13-21

46. Ribeiro dos Santos R, Marquez JO, Von Gal Furtado CC, Ramos de Oliveira JC, Martins AR, Koberle F: Antibodies against neurons in chronic Chagas' disease. Tropenmed Parasitol 1979, 30: $19-23$

47. Autoimmunity in Chagas's disease. Br Med J 1977, 2:1243-1244

48. Teixeira AR: Chagas' disease: trends in immunological research and prospects for immunoprophylaxis. Bull World Health Organ 1979, 57:697-710

49. Basombrio MA, Besuschio S: Trypanosoma cruzi culture used as vaccine to prevent chronic Chagas' disease in mice. Infect Immun 1982, 36:351-356

50. Nickell SP, Boyer MH: Trypanosoma cruzi: protective immunization of susceptible strains of mice prone to autoimmunity. Exp Parasitol 1986, 62:450-452

51. Nickell SP, Hoff R, Boyer MH: Susceptibility to acute Trypanosoma cruzi infection in autoimmune strains of mice. Parasite Immunol 1985, 7:377-386

52. Kierszenbaum F: Autoimmunity in Chagas' disease. J Parasitol 1986, 72:201-211

53. Gattass CR, Lima MT, Nobrega AF, Barcinski MA, Dos Reis GA: Do self-heart-reactive T cells expand in Trypanosoma cruzi-immune hosts? Infect Immun 1988, 56:1402-1405

54. Rizzo LV, Cunha-Neto E, Teixeira AR: Autoimmunity in Chagas' disease: immunomodulation of autoimmune and T. cruzi-specific immune responses. Mem Inst Oswaldo Cruz 1988, 83(Suppl 1): 360-362

55. Rizzo LV, Cunha-Neto E, Teixeira AR: Autoimmunity in Chagas' disease: specific inhibition of reactivity of $\mathrm{CD} 4+\mathrm{T}$ cells against myosin in mice chronically infected with Trypanosoma cruzi. Infect Immun 1989, 57:2640-2644

56. Ribeiro dos Santos R, Rossi MA, Laus JL, Silva JS, Silvino W, Mengels J: Anti-CD4 abrogates rejection and reestablishes long-term tolerance to syngeneic newborn hearts grafted in mice chronically infected with Trypanosoma cruzi. J Exp Med 1992, 175:29-39

57. McCormick TS, Rowland EC: Trypanosoma cruzi: cross-reactive anti-heart autoantibodies produced during infection in mice. Exp Parasitol 1989, 69:393-401

58. Eisen H, Kahn S: Mimicry in Trypanosoma cruzi: fantasy and reality. Curr Opin Immunol 1991, 3:507-510

59. Avila JL: Molecular mimicry between Trypanosoma cruzi and host nervous tissues. Acta Cient Venez 1992, 43:330-340

60. Davies JM: Molecular mimicry: can epitope mimicry induce autoimmune disease? Immunol Cell Biol 1997, 75:113-126 
61. Kaplan D, Vazquez M, Lafon S, Schijman AG, Levitus G, Levin MJ: The chronic presence of the parasite, and anti-P autoimmunity in Chagas disease: the Trypanosoma cruzi ribosomal $\mathrm{P}$ proteins, and their recognition by the host immune system. Biol Res 1993, 26: $273-277$

62. Cunha-Neto E, Duranti M, Gruber A, Zingales B, de Messias I, Stolf N, Bellotti G, Patarroyo ME, Pilleggi F, Kalil J: Autoimmunity in Chagas' disease cardiomyopathy: biological relevance of a cardiac myosin-specific epitope crossreactive to an immunodominant Trypanosoma cruzi antigen. Proc Natl Acad Sci U S A 1995, 92: $3541-3545$

63. Cunha-Neto E, Coelho V, Guilherme L, Fiorelli A, Stolf N, Kalil J: Autoimmunity in Chagas' disease: identification of cardiac myosinB13 Trypanosoma cruzi protein crossreactive $\mathrm{T}$ cell clones in heart lesions of a chronic Chagas' cardiomyopathy patient. J Clin Invest 1996, 98:1709-1712

64. Hernandez-Munain C, De Diego JL, Bonay P, Girones N, Fresno M: GP 50/55, a membrane antigen of Trypanosoma cruzi involved in autoimmunity and immunosuppression. Biol Res 1993, 26:209-218

65. Van Voorhis WC, Barrett L, Koelling R, Farr AG: FL-160 proteins of Trypanosoma cruzi are expressed from a multigene family and contain two distinct epitopes that mimic nervous tissues. J Exp Med 1993, 178:681-694

66. Levin MJ, Kaplan D, Ferrari I, Arteman P, Vazquez M, Panebra A: Humoral autoimmune response in Chagas' disease: trypanosoma cruzi ribosomal antigens as immunizing agents. FEMS Immunol Med Microbiol 1993, 7:205-210

67. McCormick TS, Rowland EC: Trypanosoma cruzi: recognition of a 43-kDa muscle glycoprotein by autoantibodies present during murine infection. Exp Parasitol 1993, 77:273-281

68. Bach-Elias M, Bahia D, Teixeira DC, Cicarelli RM: Presence of autoantibodies against small nuclear ribonucleoprotein epitopes in Chagas' patients' sera. Parasitol Res 1998, 84:796-799

69. Chiale PA, Rosenbaum MB, Elizari MV, Hjalmarson A, Magnusson Y, Wallukat G, Hoebeke J: High prevalence of antibodies against beta 1- and beta 2-adrenoceptors in patients with primary electrical cardiac abnormalities. J Am Coll Cardiol 1995, 26: 864-869

70. Leiros CP, Sterin-Borda L, Borda ES, Goin JC, Hosey MM: Desensitization and sequestration of human $\mathrm{m} 2$ muscarinic acetylcholine receptors by autoantibodies from patients with Chagas' disease. J Biol Chem 1997, 272:12989-12993

71. Laguens RP, Meckert PC, Chambo JG: Antiheart antibody-dependent cytotoxicity in the sera from mice chronically infected with Trypanosoma cruzi. Infect Immun 1988, 56:993-997

72. Pontes-de-Carvalho L, Santana CC, Soares MB, Oliveira GG, CunhaNeto E, Ribeiro-dos-Santos R: Experimental chronic Chagas' disease myocarditis is an autoimmune disease preventable by induction of immunological tolerance to myocardial antigens. J Autoimmun 2002, $18: 131-138$

73. Leon JS, Daniels MD, Toriello KM, Wang K, Engman DM: A cardiac myosin-specific autoimmune response is induced by immunization with Trypanosoma cruzi proteins. Infect Immun 2004, 72: $3410-3417$

74. Guinazu N, Pellegrini A, Giordanengo L, Aoki MP, Rivarola HW, Cano R, Rodrigues MM, Gea S: Immune response to a major Trypanosoma cruzi antigen, cruzipain, is differentially modulated in C57BL/6 and BALB/c mice. Microbes Infect 2004, 6:1250-1258

75. Iwai LK, Juliano MA, Juliano L, Kalil J, Cunha-Neto E: T-cell molecular mimicry in Chagas disease: identification and partial structural analysis of multiple cross-reactive epitopes between Trypanosoma cruzi B13 and cardiac myosin heavy chain. J Autoimmun 2005, 24: $111-117$

76. Sterin-Borda L, Perez Leiros C, Wald M, Cremaschi G, Borda E: Antibodies to beta 1 and beta 2 adrenoreceptors in Chagas' disease. Clin Exp Immunol 1988, 74:349-354
77. Levitus G, Hontebeyrie-Joskowicz M, Van Regenmortel MH, Levin MJ: Humoral autoimmune response to ribosomal P proteins in chronic Chagas heart disease. Clin Exp Immunol 1991, 85: $413-417$

78. Girones N, Rodriguez CI, Basso B, Bellon JM, Resino S, MunozFernandez MA, Gea S, Moretti E, Fresno M: Antibodies to an epitope from the Cha human autoantigen are markers of Chagas' disease. Clin Diagn Lab Immunol 2001, 8:1039-1043

79. Cazorla SI, Matos MN, Cerny N, Ramirez C, Alberti AS, Bivona AE, Morales C, Guzman CA, Malchiodi EL: Oral multicomponent DNA vaccine delivered by attenuated Salmonella elicited immunoprotection against American trypanosomiasis. J Infect Dis 2015, 211: 698-707

80. Guinazu N, Pellegrini A, Carrera-Silva EA, Aoki MP, Cabanillas AM, Girones N, Fresno M, Cano R, Gea S: Immunisation with a major Trypanosoma cruzi antigen promotes pro-inflammatory cytokines, nitric oxide production and increases TLR2 expression. Int J Parasitol 2007, 37:1243-1254

81. Bonney KM, Taylor JM, Daniels MD, Epting CL, Engman DM: Heat-killed Trypanosoma cruzi induces acute cardiac damage and polyantigenic autoimmunity. PLoS One 2011, 6:e14571

82. Vanderlugt CJ, Miller SD: Epitope spreading. Curr Opin Immunol $1996,8: 831-836$

83. McMahon EJ, Bailey SL, Castenada CV, Waldner H, Miller SD: Epitope spreading initiates in the CNS in two mouse models of multiple sclerosis. Nat Med 2005, 11:335-339

84. Gutierrez FR, Guedes PM, Gazzinelli RT, Silva JS: The role of parasite persistence in pathogenesis of Chagas heart disease. Parasite Immunol 2009, 31:673-685

85. Ribeiro CH, Lopez NC, Ramirez GA, Valck CE, Molina MC, Aguilar L, Rodriguez M, Maldonado I, Martinez R, Gonzalez C, Troncoso R, Lavandero S, Gingras AR, Schwaeble W, Ferreira A: Trypanosoma cruzi calreticulin: a possible role in Chagas' disease autoimmunity. Mol Immunol 2009, 46:1092-1099

86. Bonney KM, Engman DM: Chagas heart disease pathogenesis: one mechanism or many? Curr Mol Med 2008, 8:510-518

87. Girones N, Carrasco-Marin E, Cuervo H, Guerrero NA, Sanoja C, John S, Flores-Herraez R, Fernandez-Prieto L, Chico-Calero I, Salgado H, Carrion J, Fresno M: Role of Trypanosoma cruzi autoreactive T cells in the generation of cardiac pathology. Ann N Y Acad Sci 2007, 1107:434-444

88. Dumonteil E, Bottazzi ME, Zhan B, Heffernan MJ, Jones K, Valenzuela JG, Kamhawi S, Ortega J, Rosales SP, Lee BY, Bacon KM, Fleischer B, Slingsby BT, Cravioto MB, Tapia-Conyer R, Hotez PJ: Accelerating the development of a therapeutic vaccine for human Chagas disease: rationale and prospects. Expert Rev Vaccines 2012, 11:1043-1055

89. Gupta S, Garg NJ: TcVac3 induced control of Trypanosoma cruzi infection and chronic myocarditis in mice. PLoS One 2013, 8: e59434

90. Arce-Fonseca M, Ramos-Ligonio A, Lopez-Monteon A, SalgadoJimenez B, Talamas-Rohana P, Rosales-Encina JL: A DNA vaccine encoding for TcSSP4 induces protection against acute and chronic infection in experimental Chagas disease. Int J Biol Sci 2011, 7: $1230-1238$

91. Leon JS, Wang K, Engman DM: Myosin autoimmunity is not essential for cardiac inflammation in acute Chagas' disease. J Immunol 2003 , 171:4271-4277

92. Bonney KM, Gifford KM, Taylor JM, Chen CI, Engman DM: Cardiac damage induced by immunization with heat-killed Trypanosoma cruzi is not antibody mediated. Parasite Immunol 2013, 35 $1-10$

93. Lewis MD, Fortes Francisco A, Taylor MC, Burrell-Saward H, McLatchie AP, Miles MA, Kelly JM: Bioluminescence imaging of chronic Trypanosoma cruzi infections reveals tissue-specific parasite dynamics and heart disease in the absence of locally persistent infection. Cell Microbiol 2014, 16:1285-1300 
94. Goldenberg RC, Jelicks LA, Fortes FS, Weiss LM, Rocha LL, Zhao D, Carvalho AC, Spray DC, Tanowitz HB: Bone marrow cell therapy ameliorates and reverses chagasic cardiomyopathy in a mouse model. J Infect Dis 2008, 197:544-547

95. Silva DN, Souza BS, Azevedo CM, Vasconcelos JF, Carvalho RH, Soares MB, Dos Santos RR: Intramyocardial transplantation of cardiac mesenchymal stem cells reduces myocarditis in a model of chronic Chagas disease cardiomyopathy. Stem Cell Res Ther 2014, 5:81

96. Jasmin, Jelicks LA, Koba W, Tanowitz HB, Mendez-Otero R, Campos de Carvalho AC, Spray DC: Mesenchymal bone marrow cell therapy in a mouse model of Chagas disease: where do the cells go? PLoS Negl Trop Dis 2012, 6:e1971

97. Cruz-Robles D, Chavez-Gonzalez JP, Cavazos-Quero MM, PerezMendez O, Reyes PA, Vargas-Alarcon G: Association between IL-1B and IL-1RN gene polymorphisms and Chagas' disease development susceptibility. Immunol Invest 2009, 38:231-239

98. Alvarado Arnez LE, Venegas EN, Ober C, Thompson EE: Sequence variation in the IL4 gene and resistance to Trypanosoma cruzi infection in Bolivians. J Allergy Clin Immunol 2011, 127:279-282. 282.e1-3

99. Urbina JA: Recent clinical trials for the etiological treatment of chronic Chagas disease: advances, challenges and perspectives. J Eukaryot Microbiol 2015, 62:149-156

100. Eickhoff CS, Vasconcelos JR, Sullivan NL, Blazevic A, BrunaRomero O, Rodrigues MM, Hoft DF: Co-administration of a plasmid DNA encoding IL-15 improves long-term protection of a genetic vaccine against Trypanosoma cruzi. PLoS Negl Trop Dis 2011, 5: e983

101. Machado FS, Tyler KM, Brant F, Esper L, Teixeira MM, Tanowitz HB: Pathogenesis of Chagas disease: time to move on. Front Biosci 2012, 4:1743-1758

102. Araujo AF, de Oliveira G, Vasconcelos JF, Ersching J, Dominguez MR, Vasconcelos JR, Machado AV, Gazzinelli RT, Bruna-Romero O, Soares MB, Rodrigues MM: Genetic vaccination against experimental infection with myotropic parasite strains of Trypanosoma cruzi. Mediators Inflamm 2014, 2014:605023 